\title{
Buchbesprechungen-Book Reviews-Livres Nouveaux
}

P. Andreuzzi, G. C. Maggi, G. Marchetti und 5. Ragani: Das humorale Syndrom des Myocardinfarktes (La sindrome umorale dell'infarto miocardico). Edi-zioni mediche italiane, Firenze 1959.

120 Seiten umfassende Monographie, wobei die Verff. anhand 20 Beobach tungen von frischem unkompliziertem Myokardinfarkt die humoralen Blutveränderungen analysieren. Untersucht wurden: die Aktivität gewisser Enzyme (Transaminase, Aldolase, Cholesterinase, Lactohydrogenase), der Rest-N, der Blutzucker, das Haematocrit, der Blutstatus, die Cholesterolaemie, die Elektrophorese, die Reaktionen nach Baselli, Kunkel, McLagan, Wuhrmann-Wunderly, die Fibrinogenaemie, die Mucoproteinen, das aktive Globulin $\mathrm{C}$ und die Elektrolyte. Im Urin wurden die Glykocorticoiden und die 17-Ketosteroiden bestimmt. Es werden 4 Stadien des evoluierenden frischen Myocardinfarktes angegeben: I. vom 1.-2. Tag, II. vom 3.-5. Tag, III. vom 5.-10. Tag und IV. vom 10.-25. Tag. Eine diagnostische Bedeutung - bei klassischem klinischem Bild - besitzen nur wenige díeser Proben, sie erlauben doch den Verlauf des Myocardinfarktes besser zu überblicken. A. Pedrazzini, Locarno.

Buchbesprechungen - Book Reviews - Livres Nouveaux 127

Lehrbuch der speziellen pathologischen Anatomie begr. von E. Kaufmann. 11. und 12. Aufl. Hgb. von $\Lambda /$. Staemmler. III. Band, 5. Lieferung. W. de Gruy-ter \& Co., Berlin 1959.

In der vorliegenden Lieferung behandelt Peters (Bonn) die anatomíschen Veränderungen bei degenerativen Erkrankungen des Nervensystems und bei den Psychosen, sowie die traumatischen Veränderungen von Hirn und Rücken-mark. Audi diese Kapitel fügen sich sehr vorteilhaft in den Gesamtrahmen der Neuauflage dieses Lehrbuches ein, da sie auf knappem Baum umfassend und unterstützt von gut ausgewählten Abbildungen die Probleme behandeln. Ein kurzes aber gut gerichtetes Sclirifttumverzeichnis am Schlusse jedes Abschnittes erleichtert das Selbststudium einzelner Fragen. H. U. Zollinger, St. Gallen.

V. Masini: Lehrbuch der Elektrokardiographie (Compendio di elettrocardio-grafia). II Pensiero Scientifico Editore, Roma 1959. XV + 474 p.

Die Prinzipien der modernen Elektrokardiographie, das normale Elektrokardiogramm und dessen pathologische Veränderungen werden vom Verfasser an Hand zahlreicher Abbildungen besprochen. Die Unterteilung des Stoffes entspricht derjenigen der meisten diesbezüglichen Lehrbücher. Direkte Literaturhinweise im Text fehlen, doch findet sich am Schluß jeden Kapitels eine Liste der konsultierten Arbeiten. Die einzelnen Kapitel sind außerordentlich kurz und emfach gefaßt. Als Novum bringt der Verfasser am Ende der Abhandlung 200 Übungen, wobei auf der linken Seite die Diagnose, auf der rechten die EKG-Kurven abgebildet sind. Das Buch eignet sich vor allem für praktische Ärzte. A. Pedrazzini, Locarno. 
Theodor Brugsch: Kardiologie, Lehrbuch der Herz- und Gefäßkrankheiten. 5. neubearbeitete Auflage. S. Hirzel Verlag, Leipzig 1958. XII + 686 S, 183 Abb. DM 30.-. Das Buch von Brugsch erscheint in der 5. Auflage, nachdem die 1. Auflage bereits mehr als 30 Jahre zurückliegt. Charakter und ursprünglicher Aufbau wurden beibehalten, Inhalt, Text und Abbildungen entsprechend den neuen Erkenntnissen ergänzt. Das Buch ist betont klinisch konzipiert, und bei den Untersuchungsmethoden werden vor allem einfache Hilfsmittel abgehandelt, so daß das Werk in erster Linie von Studierenden («als Lehrbuch an den Universitäten und Hochschulen der Deutschen Demokratischen Republik eingeführt») gelesen werden dürfte. F. Schaub, Zurich.

F. Gigli e G. Muiesan: Semeiologia fonocardiografica. «П Pensiero Scientifico» Editore, Roma 1959. 365 p. Lire 6000.-.

Um es gleich vorwegzunehmen: dieser Leitfaden der Phonokardiographie füllt eine Lücke. Wer sich bisher in das Gebiet einarbeiten wollte, war auf Arbeiten in Zeitschriften oder auf Atlanten angewiesen und hatte Mühe, sich in der vielfältigen Terminologie zurechtzufinden.

Gigli und Muiesan lehnen sich stark an die englische Literatur, namentlich an Leatham, an. Sie gewinnen dadurch klare Vorstellungen und eindeutige Begriffe. Daneben werden Nomenklaturen und Interpretationen anderer Auto-

128 Buchbesprechungen - Book Reviews- Livres Nouveaux ren diskutiert. Das Ergebnis ist eine schöne Zusammenschau kontinentaler, englischer und amerikanischer Arbeiten.

Über die Hälfte des Buches bandelt von den verschiedenen Tönen und Ge-räuschen.

Anschließend werden die einzelnen Klappenfehler besprochen. Bei den kongenitalen

Herzfehlern wird durch die Einführung des Begriffs des Eisenmenger-Syndroms (P. Wood) eine überzeugende Vereinfachung der Klas-sierung erreicht. Der Text ist gedrängt und klar, auch für den Fremdsprachigen leicht verständlich abgefaßt. Die Abbildungen sind einheitlich beschriftet, wobei Kurven von Fallen mit unsicherer Diagnose vermieden wurden.

Es ist ein gut durchdachtes Buch, das für die Beurteilung von Phonokardiogrammen eine ausgezeichnete Grundlage liefert. Im Hinblick auf eine spätere Auflage ware die Vermehrung der Angaben von Zeitintervallen wünschenswert, z. B. Normbereich der physiologischen Tonspaltungen, Abstände der Extratöne von 1. und 2. Ton. G. Kaufmann, Zurich.

M. Facci: I toni del cuore. L. Capelli Editore, Bologna 1959. 339 p. 127 fig. Lire 5000.

Die Monographie ist ein Versuch, die phonokardiographische Analyse der Herztöne in eine bestimmte Ordnung zu bringen. Die zeitliche Beziehung zwi-schen den akustischen Phänomen und dem Ekg., den Puls- und Druckkurven wird in übersichtlichen Schemata dargestellt. Unter den unblutigen Registrie-rungsmethoden erhält das sog. Kardiogramm, die mechanische Aufzeichnung der auf die Brustwand übertragenen Herztätigkeit, besondere Berücksichtigung. Die wichtigsten Komponenten des 1. und 2. Tones werden, vor allem in Anlehnung an Luisada, als Ausdruck der Klappenschlüsse und -öffnungen interpretiert. Was im Schema überzeugend erscheint, wirkt bei der Analyse der Phonokardiogramme oft etwas spekulativ. Z. B. ergibt sich die Annahme von Aorten- und Pulmonalisöffnungstönen aus keiner der Abbildungen wirklich zwingend.

Facci geht im Dschungel der phonokardiographischen Begriffe zum Teil eigene Wege. Schwer einzusehen ist die Abgrenzung des «schiocco di chiusura e apertura delle valvule semilunari» von der gewöhnlichen Tonspaltung. Was der Autor als «tono di distensione aortica e pulmonare» 
bezeichnet, entspricht weder dem «ejection click» der Engländer noch dem $5 / 8$ Dehnungston der Deut-schen. Schließlich dient die Bezeichnung Galopp für fünf Arten von diastolischen Extratönen. Eine solche Begriffserweiterung entspricht nicht mehr der bild-haften Vorstellung eines Dreierrhythmus von bestimmter Kadenz.

Das Buch hat manche Vorzüge: klare Gliederung des Textes, gute graphische Darstellungen, ein Kapitel über fötale Herztöne und ein umfassendes Literaturverzeichnis. G. Kaufmann, Zurich. 